\title{
Conformado de piezas cerámicas a partir de suspensiones parafínicas cargadas con polvos
}

\author{
J.J. CORONEL, R. JANSSEN, N. CLAUSSEN \\ Advanced Ceramics Group, Technical University Hamburg-Harburg, Denickestrasse 15, 21073 Hamburgo, Alemania
}

\begin{abstract}
En este trabajo se demuestra la posible aplicación de suspensiones parafínicas para la formación mediante inyección o extrusión de piezas cerámicas con forma compleja. Las suspensiones fueron preparadas a partir de polvos cerámicos y metal-cerámicos. La eliminación de los componentes orgánicos así como las propiedades mecánicas después del sinterizado (resistencia a la ruptura $\sigma_{\mathrm{B}} \mathrm{y}$ tenacidad a la fractura $\mathrm{K}_{\mathrm{Ic}}$ ) también fueron evaluadas.
\end{abstract}

Palabras claves: Moldeo por inyección, extrusión, superficie específica, viscosidad, propiedades mecánicas.

\section{Shaping of ceramic parts from powder loaded wax suspensions}

In this work is demonstrated the possible application of paraffin wax suspensions for shaping complex ceramic parts by injection moulding or extrusion. These suspensions were prepared from ceramic or metal-ceramic powders. Thermal debinding as well as mechanical properties after sintering (bending strength $\sigma_{\mathrm{B}}$ and fracture toughness $\mathrm{K}_{\mathrm{IC}}$ ) were also evaluated.

Key words: injection moulding, extrusion, specific surface area, viscosity, mechanical properties.

\section{INTRODUCCIÓN}

En los últimos años el desarrollo de la tecnología para la formación por reacción de compuestos con matriz cerámica (RBAO, por sus siglas en inglés: Reaction Bonded Aluminum Oxide) ha encontrado mucho interés, debido a las ventajas en sus propiedades (mecanizado en verde, baja contracción por sinterizado, etc. $)^{1,2}$. Por otro lado, tecnologías de procesamiento de cerámicos ya establecidas como el moldeo por inyección (LPIM: Low Pressure Injection Moulding) y la extrusión han sido mejoradas constantemente ${ }^{3}$. La idea de aplicar procesos de conformado (moldeo por inyección, extrusión) a mezclas de polvos RBAO ha surgido por las mejoras que pueden ser obtenidas combinando las ventajas de ambas tecnologías (piezas con forma compleja, bajo costo, etc.). Esto se debe a que la producción de geometrías complejas mediante procesos convencionales de fabricación en Cerámicos Avanzados (por ejemplo el colaje o el prensado en seco) es posible sólo en forma limitada. Es aquí dónde tanto el moldeo por inyección como la extrusión ofrecen una alternativa. El empleo de bajas presiones y temperaturas (presión $=0.2-0.8 \mathrm{MPa}$, temperatura $=70-100^{\circ} \mathrm{C}$ ) hace al proceso muy económico en comparación con otros procesos de producción de cerámicos (por ejemplo moldeo por inyección a alta presión y temperatura). En el caso de la extrusión los costos iniciales son mayores debido a los materiales especiales y el diseño que la extrusora necesita ${ }^{4}$. El proceso permite, sin embargo, la fabricación de perfiles continuos con alto contenido en sólidos bajo las mismas condiciones que en el moldeo por inyección, incrementando al mismo tiempo la producción ${ }^{5}$. Con una adecuada optimación del sistema dispersante, se pueden emplear ambas tecnologías no sólo en la producción de piezas de geometría compleja, sino también en la fabricación de compuestos con mas de un componente. Por ejemplo, se pueden incluir partículas reforzantes no simétricas (whiskers, fibras cortas, plaquetas) con una orientación definida. Se ha demostrado ${ }^{6,7}$ que durante la extrusión tiene lugar la destrucción de aglomerados debido a los altos esfuerzos de corte en la boquilla de extrusión, como consecuencia se aumenta la homogeneidad en el producto y de esta forma se mejora la tenacidad del material.

La clave para la aplicación exitosa del conformado (moldeo por inyección o extrusión) recae en la elección de un sistema dispersante en coordinación con un adecuado proceso de eliminación del mismo ${ }^{8,9,10}$. El dispersante es el sistema orgánico, en el cuál las partículas estarán libremente dispersas. Para ajustar la composición del dispersante a un material o sistema de materiales determinado, se emplean diferentes componentes. Por ejemplo se tienen sistemas basados en polímeros rígidos y prepolímeros, basados en parafina y los basados en aceite 11,12,13,14. Estos sistemas dispersantes poseen un componente principal (parafina, polímeros termoplásticos, duroplásticos como aquellos a base de silicio) que determina las propiedades y la tenacidad del sistema; en algunos casos también un componente secundario, que modifica la viscosidad del sistema y facilita la eliminación de los componentes orgánicos (cera, aceite, termoplástico de cadena corta) y finalmente de plastificantes y surfactantes, que facilitan el conformado y desmoldeo y que ayudan a mejorar la dispersión de las partículas ${ }^{15}$.

El objetivo de este trabajo es la investigación y optimación de la fabricación de piezas cerámicas con dimensiones preci- 
sas fabricadas a partir de suspensiones parafínicas cargadas con polvos cerámicos o metal-cerámicos. De esta forma se analizarán el moldeo por inyección y la extrusión como procesos de fabricación, la influencia de los aditivos orgánicos (parafina, etc.), la eliminación de los componentes orgánicos así como del desmoldeo y se correlacionarán con las propiedades mecánicas resultantes.

\section{PROCEDIMIENTO EXPERIMENTAL.}

\subsection{Preparación de las suspensiones parafínicas.}

Los polvos metálicos y cerámicos utilizados en este trabajo se muestran en la Tabla I. El óxido de aluminio en polvo fue utilizado tal como se recibió del fabricante para la elaboración de las suspensiones cerámicas. Las mezclas RBAO para las suspensiones metal-cerámicas tuvieron la siguiente composición en volúmen: $44 \% \mathrm{Al}, 36 \% \mathrm{Al}_{2} \mathrm{O}_{3}$ y $20 \% \mathrm{ZrO}_{2}$. Estas mezclas fueron molidas en húmedo durante 3 horas utilizando ciclohexano como medio de molienda. Después de la molienda las mezclas fueron secadas a temperatura ambiente durante 24 horas y tamizadas a $200 \mu \mathrm{m}$. Para la elaboración de las suspensiones se fundió la parafina y los demás componentes orgánicos en un vaso de precipitados a una temperatura de $100{ }^{\circ} \mathrm{C}$. Acto seguido se agregaron los polvos cerámicos o metal-cerámicos según el caso y se mezcló con un agitador de vidrio. Después de esto y para mejorar la homogenización de las mezclas, éstas fueron pasadas varias veces sobre rodillos horizontales (rodillos de $\mathrm{Al}_{2} \mathrm{O}_{3}$ con un diámetro de $80 \mathrm{~mm}$ y una longitud de $200 \mathrm{~mm}$ ) hasta que se obtuvo una mezcla brillante y tersa. Más detalles sobre la composición de las suspensiones y la molienda de las mezclas se dan en otras publicaciones $^{16,17,18}$.

TABla I. MATERIALES Utilizados.

\begin{tabular}{cccccc}
\hline Material & Fabricante & Tipo & $\begin{array}{c}\text { Tamaño de } \\
\text { partícula d } \mathrm{d}_{50}[\mu \mathrm{m}]\end{array}$ & $\begin{array}{c}\text { Pureza } \\
{[\%]}\end{array}$ & $\begin{array}{c}\text { Densidad } \\
{\left[\mathrm{g} / \mathrm{cm}^{3}\right]}\end{array}$ \\
\hline $\mathrm{Al}$ & Eckart, Alemania & AS081 & 20 & $>99.5$ & 2.69 \\
$\mathrm{Al}_{2} \mathrm{O}_{3}$ & Condea, Alemania & $\mathrm{HPA} \mathrm{0,5}$ & 0.5 & $>99.5$ & 3.97 \\
$\mathrm{ZrO}_{2}$ & Z-Tech Co., USA & SFextra & 0.6 & 99.6 & 5.70 \\
Octadecilamina & Aldrich, Alemania & ODA & --- & 90 & 0.90 \\
Cera & Hoechst, Alemania & OP & --- & --- & 1.01 \\
Dispersante & Zeneca, Alemania & Solsperse 3000 & --- & --- & 0.91 \\
Parafina & Hoechst, Alemania & KX1313 & --- & --- & 0.90 \\
\hline
\end{tabular}

\subsection{Conformado.}

Mediante moldeo por inyección se prepararon probetas a partir de las suspensiones fabricadas a una temperatura de 80 ${ }^{\circ} \mathrm{C}$ y $0.4 \mathrm{MPa}$ de presión. Las dimensiones de estas muestras fueron $5 \times 6 \times 50 \mathrm{~mm}^{3}$. Se fabricaron muestras de forma compleja con propósito demostrativo (ver sección 3.2)

Utilizando una extrusora de laboratorio fabricada en la Universidad Técnica Hamburg-Harburg ${ }^{4}$ se fabricaron perfiles con una sección transversal de $5 \times 6 \mathrm{~mm}^{2}$. La fuerza y velocidad óptimas para la extrusión de las diferentes suspensiones fueron determinadas experimentalmente. La temperatura interna de la extrusora se mantuvo a $62^{\circ} \mathrm{C}$, mientras que en la zona de enfriamiento no se sobrepasáron los $40^{\circ} \mathrm{C}$.

Para la comparación de propiedades mecánicas se fabrica- ron también muestras mediante prensado en seco e isostático (50 MPa y $300 \mathrm{MPa}$ respectivamente). Las dimensiones de las probetas fueron $4 \times 5 \times 40 \mathrm{~mm}$.

\subsection{Eliminación de los componentes orgánicos.}

Previo a la oxidación (en el caso de las muestras RBAO) y posterior sinterizado de las probetas, es necesario eliminar los componentes orgánicos. Este es el paso más crítico cuando se emplea la tecnología de conformado mediante suspensiones ${ }^{17}$. Para tal efecto se empleó un procedimiento combinado de capilaridad (en una cama de polvo de $\mathrm{Al}_{2} \mathrm{O}_{3^{\prime}}$ tamaño de partícula $200 \mu \mathrm{m}$ ) y pirólisis (ver Fig. 1). Experimentos preeliminares de análisis termogravimétrico con los componentes orgánicos y con las mezclas de polvos (sólo en muestras RBAO) ayudaron a determinar las condiciones óptimas de temperatura y tiempo de permanencia en el horno.
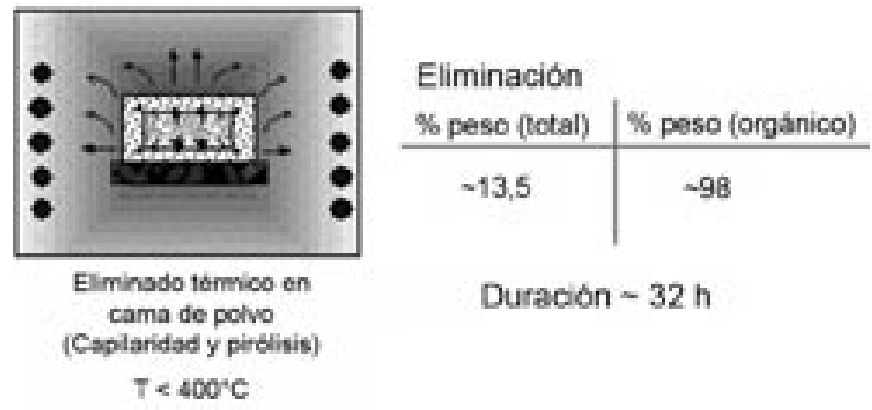

Duracion $-32 \mathrm{~h}$

Figura 1. Procedimiento de eliminación de orgánicos.

\subsection{Propiedades mecánicas.}

Después del sinterizado se evaluaron la resistencia y la tenacidad a la fractura de las diferentes probetas. Para este fin las probetas se debastaron por las cuatro caras. La cara que posteriormente estuvo sujeta a tensión fue adicionalmente pulida a $1 \mu \mathrm{m}$. Ambos experimentos fueron realizados con ayuda de una máquina universal ATS. Para la prueba de resistencia se empleó el método de flexión en cuatro puntos (bending strength) con separación entre apoyos de 10 y $20 \mathrm{~mm}$ y una velocidad de prueba de $0,5 \mathrm{~mm} / \mathrm{min}$. La resistencia $\left(\sigma_{\mathrm{B}}\right)$ se puede calcular de la siguiente manera ${ }^{19}$ :

$$
\sigma_{B}=\frac{3 F_{B}\left(S_{1}-S_{2}\right)}{2 b w^{2}}
$$

donde $\mathrm{F}_{\mathrm{B}}$ es la carga de ruptura, $\mathrm{S}_{1}$ y $\mathrm{S}_{2}$ la distancia entre apoyos, y b y w el ancho y la altura de la probeta.

La tenacidad a la fractura se evaluó mediante el método ISB (Indentation Strength in Bending). El método consiste en hacer huellas de identación Vicker con una carga determinada (en este caso $100 \mathrm{~N}$ ). A continuación se mide la resistencia restante mediante el ensayo de flexión en cuatro puntos. La tenacidad a la fractura $\left(\mathrm{K}_{\mathrm{Ic}}\right)$ se puede calcular a partir de la siguiente fórmula ${ }^{19}$ :

$$
K_{I c}=\left(\frac{256}{27} x \Psi\right)^{1 / 4}\left(\sigma_{B} P^{1 / 3}\right)^{3 / 4}
$$

donde $\mathrm{x}$ y $\Psi$ son factores numéricos $(\mathrm{x}=0,076$ y $\Psi=0,77$ en este caso), $\sigma_{\mathrm{B}}$ es la resistencia restante y $\mathrm{P}$ la carga de identación. 


\section{RESULTADOS.}

\subsection{Preparación de las suspensiones parafínicas.}

Después de la molienda se alcanzó un tamaño de partícula fino $\left(\mathrm{d}_{50}=1,5 \mu \mathrm{m}\right)$ con una distribución de tamaños relativamente ancha $\left(\mathrm{d}_{10}=0.3 \mathrm{y} \mathrm{d}_{90}=60 \mu \mathrm{m}\right)$ para las mezclas de polvos RBAO. Esta distribución de tamaños de partícula es adecuada para la elaboración de suspensiones ${ }^{3}$. Un factor muy importante lo es también la forma de partícula ${ }^{3}$. En este caso las partículas de $\mathrm{Al}$ tienen una forma bastante irregular. Esto contribuye a un incremento en las superficie específica y puede afectar negativamente la viscosidad de las suspensiones formadas ${ }^{3}$. Por otra parte existe un valor óptimo en la concentración del agente dispersante para una mezcla de polvos dada ${ }^{3}$. Este valor óptimo en la concentración satisface dos condiciones: la cantidad de agente dispersante debe ser suficiente para llenar el espacio de separación entre partículas de tal modo que éstas puedan moverse libremente con respecto a otras durante el flujo. La segunda condición es evitar la aglomeración de partículas ${ }^{7,8,12,14}$. En el caso de las suspensiones de $\mathrm{Al}_{2} \mathrm{O}_{3}$ se pudo alcanzar un máximo en el contenido de sólidos de $66 \%$ en volumen, mientras que para las suspensiones RBAO de hasta $62 \%$. Para una concentración similar dada, la dispersión de los polvos RBAO en suspensión resultó mucho más dificil de realizar que en el caso de los polvos de $\mathrm{Al}_{2} \mathrm{O}_{3}$.

\subsection{Conformado.}

Utilizando una temperatura de $80^{\circ} \mathrm{C}$ se pudieron inyectar todas las suspensiones formadas. Ejemplos de piezas formadas por inyección se muestran en las Figs. 2 y 3.

Al desmoldear las piezas pudo observarse cualitativamente que a mayor cantidad de sólidos en suspensión se hizo más fácil el desmoldeo. Comparativamente las suspensiones RBAO presentaron incluso una menor adherencia a las paredes de los moldes empleados. Una posible explicación a éste fenómeno es dada por ${ }^{21}$. La fricción con las paredes de un molde tiene influencia en el comportamiento en flujo para mezclas de polímeros y cerámicos durante la inyección. La fricción a su vez puede verse afectada por los aditivos orgánicos utilizados. Los grupos polares presentes en el sistema dispersante tienen cierta preferencia con grupos polares en la pared del molde y no tanto así con la masa polímerica no polar. Parte del dispersante será adsorbido en los grupos polares de la pared del molde y esto puede reducir la fricción entre las partículas y la pared. En el caso de las suspensiones fabricadas en este trabajo se tiene que a mayor contenido de sólidos, se tendrán menos moléculas de dispersante "libres" para adherirse a las paredes del molde. Comparativamente la irregularidad y la mayor superficie específica de las partículas de Al hacen que éstas adsorban más dispersante que las partículas cerámicas, por lo que se tendrán aun menos moléculas dispersantes disponibles para adherirse a la pared del molde. En consecuencia se tiene una menor adherencia. Esta misma explicación acerca de la adherencia, aclararía también porque la carga necesaria para extruir una suspensión determinada disminuye conforme aumenta el contenido de sólidos en suspensión y a su vez es menor para suspensiones RBAO que para aquellas de $\mathrm{Al}_{2} \mathrm{O}_{3}$ (ver Fig. 4). Ejemplos de piezas extruídas se dan en la Figura 5.

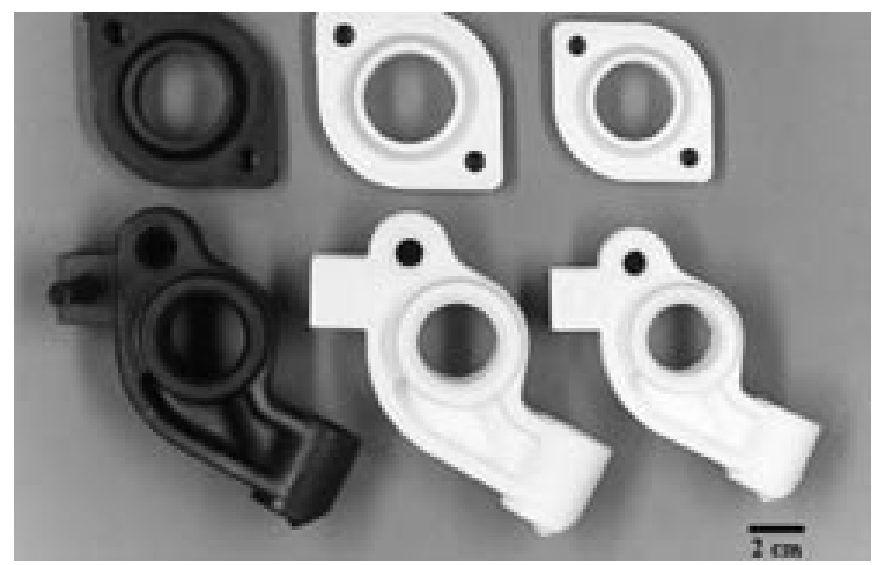

Figura 2. Ejemplo de probetas fabricadas por inyección: Cojinetes y válvulas en verde, después de la oxidación y después del sinteriza$\mathrm{do}^{20}$.

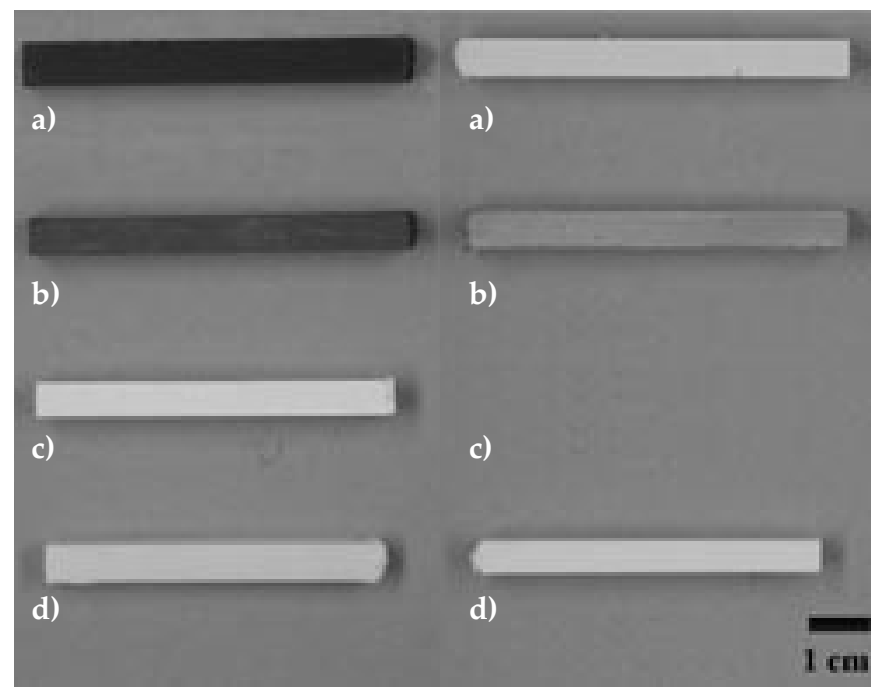

Figura 3. Ejemplo de probetas fabricadas por inyección: Probetas en verde (a), después del eliminado térmico (b), después de la oxidación -sólo RBAO- (c) y después del sinterizado (d). ${ }^{20}$

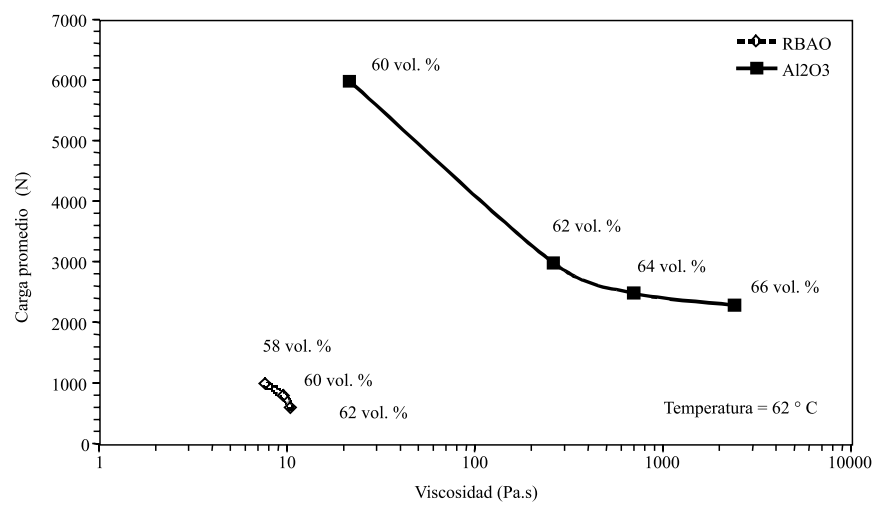

Figura 4. Carga promedio necesaria para la extrusión de las diferentes suspensiones (adaptado de ${ }^{18}$ ). 


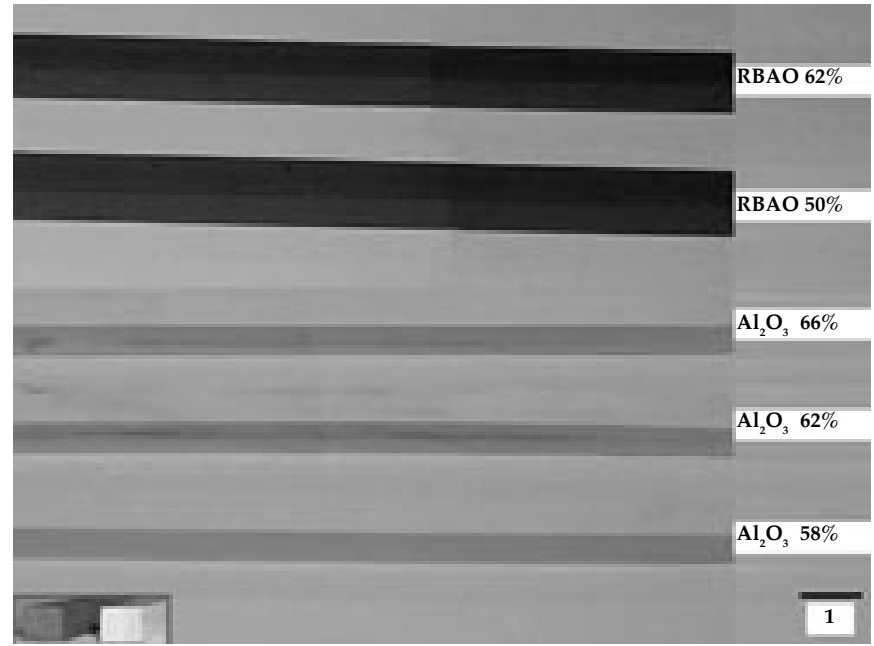

Figura 5. Ejemplo de probetas fabricadas por extrusión ${ }^{20}$.

\subsection{Eliminación de los componentes orgánicos.}

La Fig. 6 muestra las curvas del análisis termogravimétrico del sistema dispersante y sus componentes individuales. Hasta una temperatura de $190^{\circ} \mathrm{C}$ se mantienen los componentes orgánicos prácticamente intactos. A partir de esta temperatura comienza la evaporación y a temperaturas más elevadas la descomposición térmica.

En el caso de los polvos RBAO utilizados, la oxidación del $\mathrm{Al}$ comienza aproximadamente a $260^{\circ} \mathrm{C}$ (ver Fig. 7). Con base en estos datos se estableció un ciclo de eliminación de orgánicos en tres fases (las suspensiones RBAO fueron tomadas como base para este ciclo debido a que éstas se mostraron más susceptibles a la aparición de grietas durante la eliminación de los componentes orgánicos). La primera fase consistió en un tiempo de permanencia a $100^{\circ} \mathrm{C}$ para permitir la extracción mediante capilaridad a través de la cama de polvos de $\mathrm{Al}_{2} \mathrm{O}_{3}$. La segunda etapa consistió en un tiempo de permanencia a $190{ }^{\circ} \mathrm{C}$ durante el cuál tuvo lugar tanto la capilaridad como la evaporación de los componentes orgánicos. Finalmente una tercera etapa consistente de un tiempo de permanencia a $330^{\circ} \mathrm{C}$ dónde se produjeron tanto la oxidación del $\mathrm{Al}$ y la descomposición térmica de los componentes orgánicos. Los tiempos de permanencia a las diferentes temperaturas fueron ajustados experimentalmente para cada suspensión y sección transversal de las probetas fabricadas para tal efecto. La Fig. 8 muestra el ciclo de eliminación térmica correspondiente a probetas RBAO fabricadas con una suspension conteniendo un $58 \%$ en volumen de sólidos y una sección transversal de $5 \times 6 \mathrm{~mm}^{2}$.

Es importante hacer notar que la primera etapa de la eliminación de orgánicos es muy importante, ya que en ella se tiene que generar suficiente porosidad en el material para permitir la salida de los gases generados por la descomposición de los componentes orgánicos sin riesgo de formación de grietas en el material.

\subsection{Propiedades mecánicas.}

Las Tablas 2 y 3 muestran la resistencia y la tenacidad a la fractura después del sinterizado para las diferentes suspensiones utilizadas.

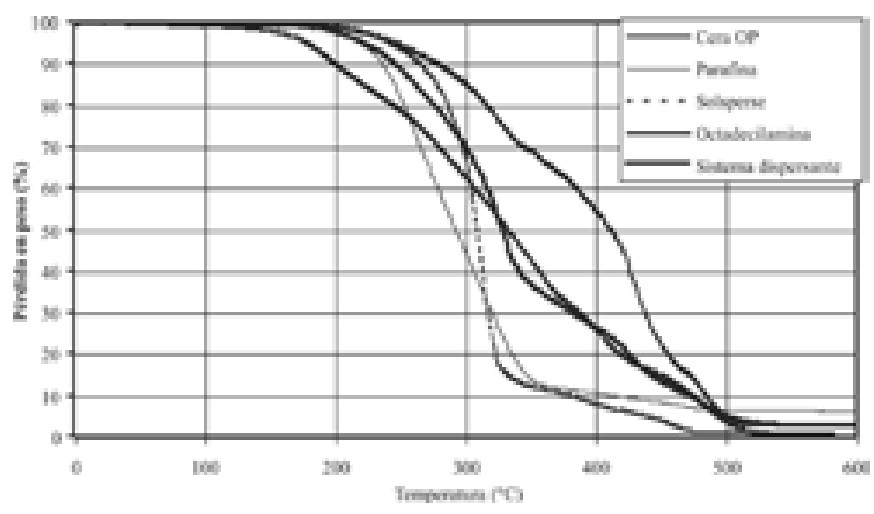

Figura 6. Análisis termogravimétrico para el sistema dispersante y los componentes individuales (adaptado de ${ }^{18}$ ).

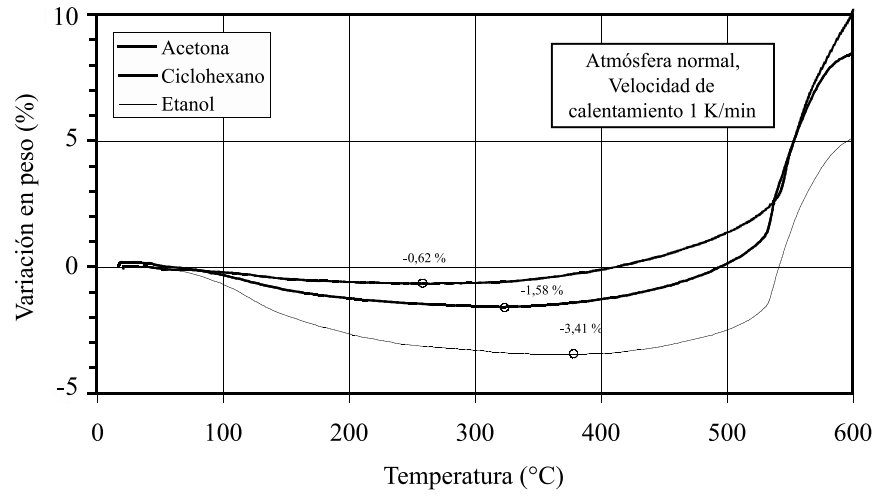

Figura 7. Análisis termogravimétrico para mezclas de polvos RBAO molidos en diferentes medios ${ }^{20}$.

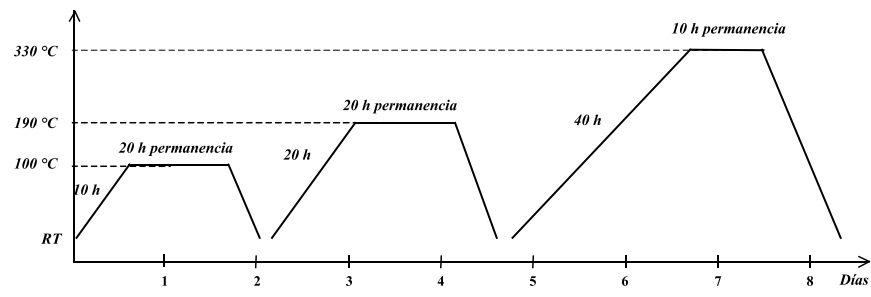

Figura 8. Ciclo de elimación térmica de orgánicos.

Tabla II. Resistencia a la fractura (MPa). Prueba De Flexión eN CUATRO PUNTOS, DISTANCIA ENTRE APOYOS 10 Y $20 \mathrm{MM}$.

\begin{tabular}{|c|c|c|c|c|}
\hline \multirow{2}{*}{ Suspensión } & $\begin{array}{c}\text { Contenido de } \\
\text { sólidos (Vol.-\%) }\end{array}$ & Inyección & Extrusión & $\begin{array}{c}\text { Prensado } \\
\text { isostático en frío }\end{array}$ \\
\hline \multirow{3}{*}{$\mathrm{RBAO}$} & 58 & $186 \pm 25$ & $220 \pm 20$ & \\
\cline { 2 - 4 } & 60 & $221 \pm 15$ & $180 \pm 11$ & $400 \pm 64$ \\
\cline { 2 - 4 } & 62 & $240 \pm 27$ & $190 \pm 17$ & \\
\hline \multirow{3}{*}{$\mathrm{Al}_{2} \mathrm{O}_{3}$} & 60 & $185 \pm 19$ & $170 \pm 20$ & \\
\cline { 2 - 4 } & 62 & $170 \pm 12$ & $160 \pm 16$ & \multirow{2}{*}{$370 \pm 58$} \\
\cline { 2 - 4 } & 64 & $255 \pm 10$ & $179 \pm 18$ & \\
\cline { 2 - 4 } & 66 & $269 \pm 46$ & $235 \pm 23$ & \multicolumn{1}{|c}{} \\
\hline
\end{tabular}


Tabla III. Tenacidad a la Fractura (MPA*M1/2). Prueba De FleXión EN CUATRO PUNTOS CON PREVIA IDENTACIÓN VICKER'S EN EL MATERIAL (100 $\mathrm{N}$ DURANTE $20 \mathrm{~s})$.

\begin{tabular}{|c|c|c|c|c|}
\hline Suspensión & $\begin{array}{c}\text { Conenido en } \\
\text { sólidos (Vol.- } \% \text { ) }\end{array}$ & Inyección & Extrusión & $\begin{array}{c}\text { Prensado } \\
\text { isostático en frío }\end{array}$ \\
\hline \multirow{3}{*}{ RBAO } & 58 & $3,4 \pm 0,3$ & $3,9 \pm 0,2$ & \\
\cline { 2 - 5 } & 60 & $3,6 \pm 0,2$ & $3,6 \pm 0,1$ & $4,1 \pm 0,1$ \\
\cline { 2 - 5 } & 62 & $3,9 \pm 0,1$ & $3,5 \pm 0,3$ & \\
\hline \multirow{3}{*}{$\mathrm{Al}_{2} \mathrm{O}_{3}$} & 60 & $2,8 \pm 0,1$ & $3,2 \pm 0,4$ & \\
\cline { 2 - 4 } & 62 & $3,2 \pm 0,1$ & $3,2 \pm 0,3$ & \multirow{2}{*}{$3,9 \pm 0,3$} \\
\cline { 2 - 4 } & 64 & $3,4 \pm 0,1$ & $3,2 \pm 0,2$ & \\
\cline { 2 - 5 } & 66 & $3,5 \pm 0,1$ & $3,2 \pm 0,2$ & \\
\hline
\end{tabular}

En el caso de las muestras producidas por inyección tanto $\sigma_{\mathrm{B}}$ como $\mathrm{K}_{\mathrm{IC}}$ aumentan con el contenido de sólidos en la suspensión original. En el caso de las probetas fabricadas por extrusión no se pudo observar una clara tendencia. Durante el proceso de extrusión el aire o los componentes orgánicos atrapados entre partículas generarían defectos al no tener salida libre durante el proceso de eliminación de orgánicos. Este tipo de defectos, aunque de tamaño microscópico, no pueden ser compensados por sinterización y afectan negativamente la resistencia a la ruptura del material ${ }^{20}$.

A partir de los datos de las propiedades mecánicas y empleando la ecuación de Griffith (3) puede calcularse el tamaño crítico de defectos $a$ en las diferentes piezas cerámicas fabricadas a partir de las suspensiones (ver Figura 9).

$$
\begin{gathered}
\sigma_{B}=\frac{1}{Y} \cdot \frac{K_{I C}}{\sqrt{a}} \quad \operatorname{con} Y=\sqrt{\pi} \\
y \quad a=\frac{1}{\pi} \cdot\left(\frac{K_{I C}}{\sigma_{B}}\right)^{2}
\end{gathered}
$$

Esta gráfica muestra que el defecto máximo que se puede encontrar en una muestra producida por prensado isostático es menor que aquel en el caso de muestras producidas por inyección o extrusión. Esto explica también la mayor resistencia a la fractura en estas muestras.

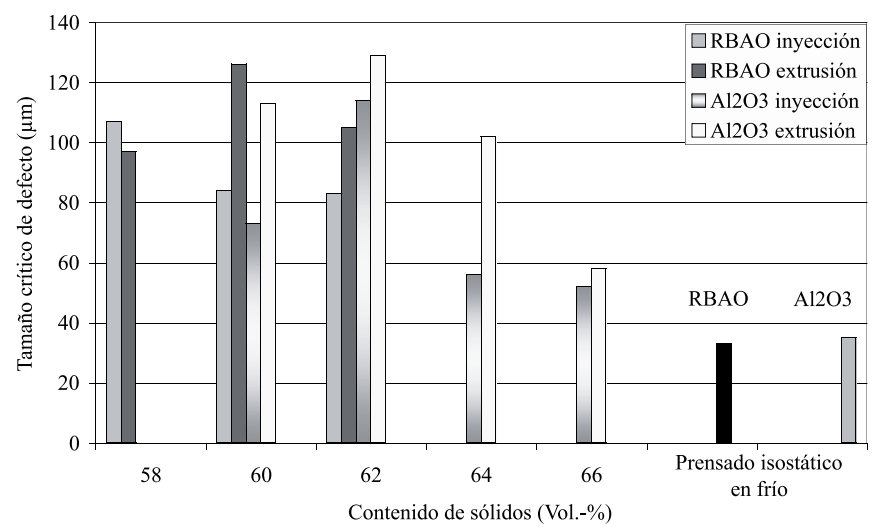

Figura 9. Tamaño típico de defectos $a$.

\section{CONCLUSIONES}

En este trabajo se ha mostrado la posibilidad de la fabricación de suspensiones parafínicas con un alto contenido de sólidos en volumen (hasta un $66 \%$ en el caso de polvos cerámicos y hasta $62 \%$ para polvos metal-cerámicos). Con las suspensiones asi producidas fue posible la fabricación de probetas y piezas modelo mediante inyección o extrusión.

El proceso térmico de eliminación de los componentes orgánicos necesita ser mejorado considerablemente, ya que hasta el momento se requieren tiempos muy largos para garantizar la fabricación de piezas libres de defectos, principalmente en el caso de suspensiones con polvos metal-cerámicos.

Las propiedades mecánicas señalan una tendencia de mejoramiento con el incremento de sólidos en la suspensión original. Las propiedades mecánicas de muestras prensadas isostáticamente son mejores, pero el grado de complejidad que se puede lograr en las piezas formadas por el moldeo por inyección o por extrusión es una característica muy atractiva de esta tecnología.

\section{AGRADECIMIENTOS.}

Este trabajo se ha realizado dentro del proyecto E.508.032 DFG (Sociedad Alemana de investigación). También se recibio ayuda del DAAD (Servicio Alemán de Intercambio Académico) y del CONACyT (Consejo Nacional de Ciencia y Tecnología - México).

\section{BIBLIOGRAFÍA.}

1.- Claussen N., Le T., Wu S. "Low-Shrinkage Reaction-Bonded Alumina." J. Eur. Ceram. Soc. 5 29-35 (1989)

2.- Holz D. "Herstellung und Charakterisierung von reaktionsgebundenen $\mathrm{Al}_{2} \mathrm{O}_{3}$-Keramiken (RBAO-Verfahren) am Beispiel des Systems $\mathrm{Al}_{2} \mathrm{O}_{3} / \mathrm{ZrO}_{2}$." Fortschr.-Ber. Düsseldorf: VDI-Verlag. 5 [367] (1994).

3.- German R. "Powder Injection Molding." Metal Powder Industries Federation, Princeton (1990).

4.- Schneider T.A. "Konzeption eines Laborextruders." Diplomarbeit, Advanced Cramics Group, Technical University Hamburg-Harburg (1998).

5.- Crumm A.T., Halloran J.W. "Fabrication of Microconfigured Multicomponent Ceramics.“ J. Am. Ceram. Soc., 78 [5] 1313-23 (1998)

6.- Wildman R.D., Blackburn S. "Breakdown in Agglomerates in Ideal Pastes During Extrusion.“ J. Mat. Sci. 33 5119-124 (1998).

7.- Bergström L. "Rheological Properties of Concentrated Nonaqueous Silicon Nitride Suspensions.“ J. Am. Ceram. Soc., 79 [12] 3033-3040 (1996).

8.- Benbow J.J., Blackburn S., Mills H. "The Effects of Liquid-Phase Rheology on the Extrusion Behaviour of Paste.“ J. Mat. Sci., 33, 5827-5833 (1998).

9.- Vandeneede V., Moortgart G., Cambier F. “Characterisation of Alumina Pastes for Plastic Moulding.“ J. Eur. Ceram. Soc. 17 225-231 (1997).

10.- Richerson D. "Modern Ceramic Engineering." Marcel Dekker, Inc., New York (1982).

11.- Wegmann M., Gut B., Barroth K. “Extrusion of Polycrystalline Ceramic Fibers." cfi/Ber. DKG, 75 [10] 35-37 (1998).

12.- Evans J.R. "Plastics Technology Applied to Ceramic Suspensions.“ DKG, 8 81-106 (1993).

13.- Novak S., Vidovic K., Sajko M., Kosmac T. “Surface Modification of Alumina Powder for LPIM.“ J. Europ. Ceram. Soc. 17 217-223 (1997).

14.- Bergström L., Schinozaki K., Tomiyana H., Mizuta N. “Colloidal Processing of a very fine $\mathrm{BaTiO}_{3}$ Powder, Effect of Particle Interactions on the Suspension Properties, Consolidation und Sintering Behaviour." J. Am. Ceram. Soc., 80 [2] 291-300 (1996).

15.- Mangels J., Trela W. “Ceramic Components by Injection Molding.“ Advances in Ceramics, The American Ceramic Society, Inc., Columbus. 9 220-233 (1984).

16.- Leverköhne M. “Fabrication and Characterization of Multifunctional MetalCeramic Composites." Fortschr.-Ber. Düsseldorf: VDI-Verlag. VDI Reihe 5 Nr. 637 (2001). 
17.- Janssen R., Leverkoehne M., Coronel J.J. "Multifunctional Metal-Ceramic Composites by Solid Free Forming (SFF)". To be published at: Ceram. Eng. Sci. Proc. (2004).

18.- Leverkoehne M., Coronel J.J., Dirscherl R., Gorlov I., Janssen R., Claussen N. “Novel Binder System Based on Paraffin-Wax for Low-Pressure Injection Molding of Metal-Ceramic Powder Mixtures". Ad. Eng. Mat. 3 [12] 2001, 995-998.

19.- Scheppokat S. "Reaktionsgebundener Mullit mit Nullschrumpfung auf Basis des Precursorsystems $\mathrm{Al} / \mathrm{Al}_{2} \mathrm{O}_{3} / \mathrm{SiC}$. Fortschr.-Ber. Düsseldorf: VDIVerlag. 5 [552] (1999).

20.- Coronel J.J. “Endkonturnahe Formgebung keramischer Bauteile über pul- verbeladene Wachssuspensionen". Ph.D. Thesis. Technical University Hamburg-Harburg. Germany, 2004.

21.- Lindqvist K., Carlström E., Persson M., Carlsson R. “Organic Silanes and Titanates as Processing Additives for Injection Molding of Ceramics." J. Am. Ceram. Soc. 72 [1] 99-103 (1989).

22.- Coronel J.J., Leverkoehne M., Janssen R. and Claussen N. "Design of Paraffin Suspensions Highly Loaded with Ceramic or Metal-Ceramic Powders". Ceram. Eng. Sci. Proc. 22 [4] 67-74 (2001).

Recibido: 06.02.04

Aceptado: 11.05.04 